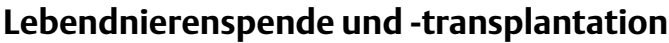 \\ Hohe Transplantatüberlebensraten bei kalkulierbarem Risiko
}

\author{
A. Voiculescu' ${ }^{1}$ K. Ivens ${ }^{1}$, W. Sandmann², B. Grabensee ${ }^{1}$ \\ ${ }^{1}$ Klinik für Nephrologie und Rheumatologie, Medizinische Klinik und Poliklinik, Universitätsklinikum Düsseldorf \\ (Direktor: Prof. Dr. B. Grabensee) \\ ${ }^{2}$ Klinik für Gefäßchirurgie und Nierentransplantation, Medizinische Klinik und Poliklinik, Universitätsklinikum Düsseldorf \\ (Direktor: Prof. Dr. W. Sandmann)
}

$\mathrm{B}$ ereits in den 80er Jahren wurde sowohl in angloamerikanischen als auch in skandinavischen Ländern die Lebendspendetransplantation auch zwischen nicht Blutsverwandten immer häufiger durchgeführt. Im letzten Jahr hat in den USA die Zahl der Lebendnierentransplantationen die Zahl der Leichennierentransplantationen übertroffen, das Verhältnis betrug etwa 54 zu 46\%. In Mitteleuropa lag der Anteil der Nierentransplantationen mit Organen von Lebendspendern noch bis 1995 ungefähr bei $5 \%$.

Trotz fehlender HLA-Kompatibilität (HLA = „human leukocyte antigen“) waren die Patienten- und Transplantatüberlebensraten nach Lebendnierentransplantationen zwischen nichtverwandten Personen günstiger als nach postmortaler Nierentransplantation $(11,23)$. Daher - und weil nur wenig Organe von postmortalen Spendern zur Verfügung stehen - sind heute immer mehr Lebendnierenspenden und -transplantationen zu verzeichnen. Außerdem belegen Kurz- und Langzeitdaten kalkulierbare Risiken für die Spender, was diesen die Entscheidung zu einer Lebendnierenspende erleichtert (17). Auch in Deutschland hat die Zahl der Lebendnierentransplantationen stetig

In Anbetracht von niedrigen und stagnierenden Zahlen von postmortalen Nierenspenden, der geregelten Rechtslage und der guten Ergebnisse der Lebendspende-Nierentransplantationen, hat die Zahl dieser auch in Deutschland in den letzten Jahren deutlich zugenommen. Als Spender kommen verwandte und nichtverwandte Personen infrage, die dem Empfänger in besonderer persönlicher Verbundenheit offenkundig nahe stehen. Neben Freiwilligkeit und Ausschluss von Organhandel sind die Blutgruppenkompatibilität, ein negatives Crossmatch und der Ausschluss von schwer wiegenden Erkrankungen Voraussetzungen zur Lebendnierenspende und -transplantation. Psychologische und ethische Faktoren müssen ebenfalls berücksichtigt werden. Die Vorteile der Lebendnierenspende und -transplantation liegen in der Planbarkeit mit Schaffung optimaler Voraussetzungen und in den exzellenten Transplantatüberlebensraten, sowohl nach Spenden zwischen Verwandten als auch zwischen Nichtverwandten. Die Risiken für den Spender sind kalkulierbar, aber nicht zu vernachlässigen, und eine detaillierte Aufklärung ist Voraussetzung. Die Förderung der Lebendnierenspendetransplantation unter anderem durch die häufigere Durchführung von präemptiven Transplantationen oder dem Einschluss älterer Spender - darf aber die Bemühungen bezüglich der Leichennierenspende nicht in den Hintergrund rücken lassen.

zugenommen seit das Transplantationsgesetz 1997 in Kraft getreten ist: 2002 waren dies immerhin 19\% aller Nierentransplantationen in Deutschland (Abb. 1) (5).

\section{Rechtliche Voraussetzungen}

In Deutschland regelt der dritte Abschnitt des Transplantationsgesetzes sowohl die Spende, die Entnahme als auch die Übertragung von Organen bei lebenden Organspendern. Hier wird unter anderem die Subsidiarität der Lebendspende festgelegt. Die Entnahme eines nichtregenerierbaren Organs beim lebenden Spender ist demnach nur dann zulässig, wenn kein geeignetes postmortal entnommenes Organ zur Verfügung steht. Darüber hinaus darf eine solche Intervention nur erfolgen, wenn der Spender ein Verwandter ersten oder zweiten Grades, der Ehegatte, der Verlobte oder eine andere Personen ist, die dem Empfänger in besonderer persönlicher Verbundenheit offenkundig nahe steht.

Die Entnahme darf erst erfolgen, nachdem sich Organspender und -empfänger zur Teilnahme an einer ärztlich empfohlenen Nachbetreuung bereit erklärt haben. Eine weitere Voraussetzung ist, dass eine 
nach Landesrecht zuständige Kommission gutachterlich dazu Stellung genommen hat, ob begründete tatsächliche Anhaltspunkte dafür vorliegen, dass die Einwilligung in die Organspende nicht freiwillig erfolgt oder das Organ Gegenstand verbotenen Handeltreibens ist (Transplantationsgesetz).

Die Lebendspende wird in den deutschsprachigen Ländern unterschiedlich geregelt. So wird in Deutschland eine Transplantation unter Verwandten und zwischen Ehepartnern oder Lebensgefährten durchgeführt. Gefordert wird eine mehrjährige Beziehung bzw. Bekanntschaft und/oder eine bereits seit längerem bestehende Lebensgemeinschaft. Demzufolge sind in Deutschland bislang noch keine so genannten Crossover-Transplantationen (z.B. zwischen zwei Ehepaaren, bei denen aufgrund fehlender
Blutgruppenübereinstimmung eine direkte Transplantation unter den Ehepartnern nicht möglich ist) durchgeführt worden. Momentan steht allerdings zur Diskussion, inwiefern eine „besondere persönliche Verbundenheit" nicht auch im Rahmen einer Vorbereitung zu einer solchen Crossover-Transplantation entstehen kann. In der Schweiz ist diese Form der Transplantation ebenso möglich wie in Österreich. In Österreich wird insgesamt die Lebendspendetransplantation großzügiger gehandhabt.

\section{Medizinische \\ Voraussetzungen}

Grundvoraussetzung einer jeden Nierenspende ist die Blutgruppenkompatibilität (gleiche Blutgruppen oder Blutgruppe 0 als Universalspender). Der Rhesus-Faktor spielt keine Rolle. Untersuchungen auf

\section{Abb. 1 Anteil der Nierenlebendspenden an der Nierentransplantation in Deutschland}

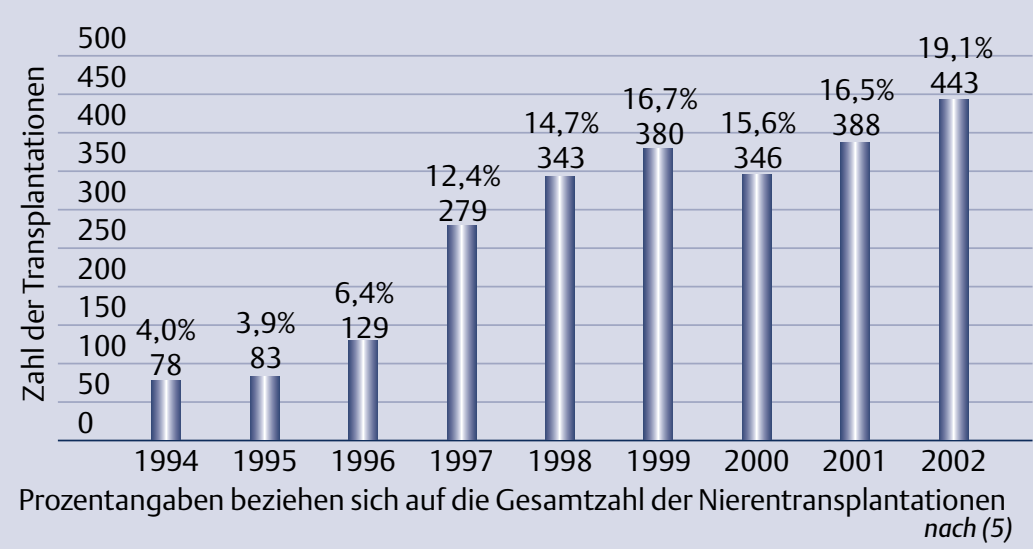

\section{Abb. 2 Transplantatüberleben von Lebendspendetransplantationen}

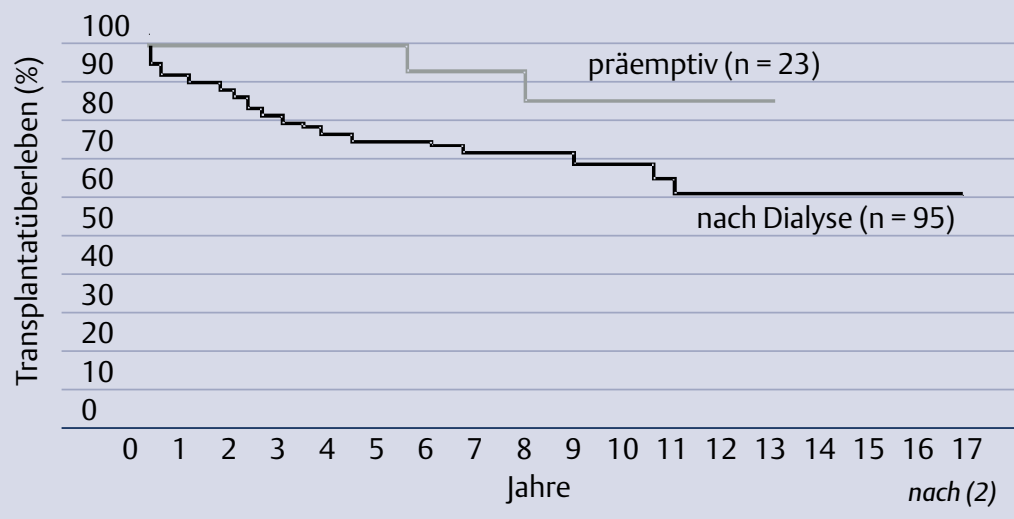

HLA-Kompatibilität werden immer durchgeführt - obwohl auch eine komplette Missmatchkonstellation, wie sie bei Nichtverwandten häufig vorliegt, keine Kontraindikation ist. Das Cross-Match muss jedoch negativ sein.

Dem Spender, der keinen gesundheitlichen Vorteil zu erwarten hat, ist nur ein geringes unvermeidbares Restrisiko zuzumuten. Erkrankungen, die zur deutlichen Erhöhung des Narkoserisikos führen (z.B. eine schwere koronare Herzkrankheit oder eine deutliche Lungenfunktionseinschränkung), gelten in der Regel als Kontraindikationen für eine Spende. Die Kreatininclearance sollte innerhalb der Standardabweichungen der altersadaptierten Normwerte liegen. Hat eine Niere eindeutig einen geringeren Anteil an der Gesamtfunktion, entscheidet man sich bei der Transplantation für dieses Organ. Bei Proteinurie und anderen Auffälligkeiten im Urinsediment sind ausführliche urologische und nephrologische Abklärungen (inklusive einer Nierenbiopsie) gerechtfertigt - sowohl zur Abklärung für den Spender selbst, wie auch zur Prüfung der Spendereignung.

Malignome gelten prinzipiell als Ausschlusskriterium für eine Nierenspende. Die Übertragung von Infektionskrankheiten viraler und bakterieller Art werden detailliert betrachtet, und eine Infektion über die Nierenspende muss vermieden werden. In Bezug auf das Spenderalter sind keine festen Grenzen nach oben gesetzt: Dieses bleibt eine individuelle Entscheidung in Abhängigkeit vom Allgemeinzustand des potenziellen Spenders.

Letztendlich werden nach einer ausführlichen Voruntersuchung lediglich $30-50 \%$ der sich initial vorstellenden Paare tatsächlich auch zur Spende und Transplantation zugelassen $(21,25)$. Gründe, die in unserem Zentrum letztendlich gegen eine Spende und Transplantation sprachen, fasst die Tabelle 2 zusammen.

All dies erklärt den enormen personellen und finanziellen Aufwand und führt nicht selten zu Frustrationen und Unzufriedenheit bei den Patienten und ihren potenziellen Spendern. Häufig sind Ableh- 
nungen aus medizinischen und psychologischen Gründen ein Anlass für das Spender-Empfänger-Paar, sich in einem anderen Zentrum erneut vorzustellen.

\section{Psychologische und ethische Konflikte}

Die psychologische Evaluation ist fester Bestandteil in der Vorbereitung zur Lebendnierenspende bzw. zur Transplantation. Entscheidend ist hierbei sicherlich der Ausschluss psychischer Erkrankungen, die eine Gefährdung für den Spender oder Empfänger darstellen können oder solche, welche die Verarbeitung schwieriger Situationen während des Spende- und Transplantationsprozesses verhindern würden.

Deutlich schwieriger ist es, die Freiwilligkeit der Spende zu beurteilen. So kann sowohl unter Verwandten als auch unter Nichtverwandten der innere Druck zur Lebensrettung eines Angehörigen oder Freundes erheblich sein. Emotionale, familiäre und finanzielle Abhängigkeitsverhältnisse sind ebenfalls nicht immer $\mathrm{zu}$ durchschauen. Eine endgültige Beurteilung gelingt nur, wenn die betreuenden Nephrologen, die Mitglieder der Transplantationszentren und die Psychologen/Psychiater den Fall gemeinsam evaluieren. Zum Schluss wird der Spender bei der nach Landesrecht eingerichteten Kommission vorgestellt, die meist bei der Ärztekammer angesiedelt ist. Diese Kommission nimmt gutachterlich Stellung, ob begründete Anhaltspunkte dafür vorliegen, dass die Einwilligung zur Organspende nicht freiwillig erfolgt. Die Stellungnahme der Kommission ist nicht bindend, wird aber von uns stets akzeptiert.

\section{Vorteile der Lebendnieren- transplantation}

Die Vorteile einer Lebendnierenspende und -transplantation sind zum einen die Planbarkeit des Eingriffes und die Möglichkeit, eine immunsuppressive Medikation bereits frühzeitig einleiten zu können. Außerdem ist in diesem Fall eine präemptive Transplantation möglich, sodass eine Hämodialyse- bzw. Peritonealdialysetherapie nicht nötig wird, was zu noch besseren Ergebnissen geführt hat (Abb. 2.) $(2,12,16)$. Ein weiterer Vorteil ist, dass eine kurze kalte Ischämiezeit eingehalten werden kann. Denn so bleibt der Reperfusionsschaden, der mit einer erheblichen Zytokinfreisetzung einhergeht und damit Abstoßungsreaktionen auslösen kann, möglichst gering.

Zudem ist bei der Lebendnierenspende die Expression von HLA-Antigenen und Adhäsionsmolekülen - anders als bei der Leichennierenspende - deutlich reduziert. Bei der Leichennierenspende scheint der Hirntod für die hohe Expressionsrate dieser Substanzen verantwortlich zu sein (13). Ein positiver Einfluss auf die Beziehung zwischen Spender und Empfänger wurde ebenfalls festgestellt. Die Compliance bezüglich der regelmäßigen Medikamenteneinnahme war bei Patienten mit nichtverwandten Lebendtransplantationen besonders hoch (13).

\section{Durchführung von Spende und Transplantation}

Vor der Transplantation muss der Empfänger nach den üblichen Richtlinien zur Klärung der Transplantabilität vorbereitet und in die Warteliste zur Transplantation bei Eurotransplant (ET) aufgenommen werden. Je nach Therapiekonzept des Zentrums beginnt dann der Empfänger - beispielsweise fünf Tage vor der Transplantation - mit der Einnahme von Immunsuppressiva (z.B. Mycophenolatmofetil, Cell Cept ${ }^{\circledR}$; Cyclosporin A, Sandimmun optoral ${ }^{\circledR}$ oder Tacrolimus, Prograf ${ }^{\circledR}$ ). Am Tag der Transplantation erhält er dann zusätzlich Steroide (z.B. Soludecortin ${ }^{\circledR}$ ) und bei mehr als vier Missmatches oder Immunisierung auch mono- oder polyklonale Antikörper (Muromonab-CD3, Orthoclone ${ }^{\circledR}$ OKT3; Antithymozyenglobulin, ATG-Fresenius $\left.{ }^{\circledR}\right)$ oder Interleukin-RezeptorAntagonisten (Basiliximab, Simulect $^{\circledR}$; Daclizumab, Zenapax $\left.{ }^{\circledR}\right)$.

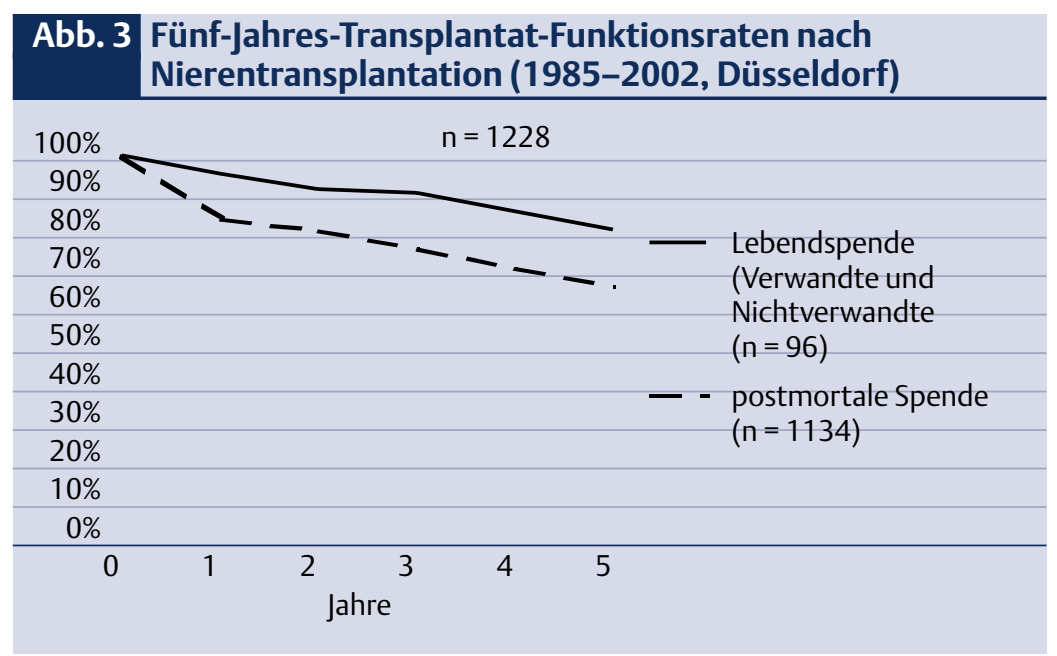

\section{Tab. 1 Obligate Untersuchungen bei potenziellen Nierenspendern}

\begin{tabular}{ll} 
& Untersuchungen \\
\hline Labor & Blutgruppe, HLA-Typisierung, Cross-Match \\
& Virusserologie (Hepatitis A, B und C; HIV; Zytomegalie, EBV) \\
& Routinelabor, Kollagenose-Parameter \\
\hline Urin & Status/Sediment, Mikrobiologie \\
& Kreatininclearance, Proteinurie, SDS-Page \\
\hline apparative Untersuchungen & EKG mit Belastung, Röntgen-Thorax, Echokardiografie \\
& 24-Stunden- Blutdruckmessung \\
& Abdomensonografie, Nierenfunktionsszintigrafie \\
& Angiografie mit Spätphase (MRA, CT-Angio) \\
& zur Darstellung der ableitenden Harnwege \\
\hline konsiliarische Untersuchungen & psychologische/psychiatrische Evaluation \\
\hline
\end{tabular}


Im Verlauf wird die Immunsuppression reduziert: In der Regel kann der Transplantatempfänger nach einem halben Jahr mit stabiler Transplantatfunktion auf eine ZweifachMedikation umgestellt werden, um die Langzeitnebenwirkungen der Immunsuppressiva möglichst gering zu halten. Als Abstoßungstherapien werden hoch dosiert Steroide oder monoklonale Antikörper verabreicht (in Abhängigkeit von der Art und Schwere der Abstoßung).

Die Operation selbst erfolgt meist in parallelen Operationssälen, um die kalte Ischämiezeit auf ein Minimum reduzieren zu können. Entnommen wird die Niere über einen extraperitonealen Flankenschnitt. Eine Alternative ist beispielsweise ein anteriorer Zugang, der ebenfalls extraperitoneal durchgeführt wird.
Immer häufiger werden mit gutem Erfolg auch laparoskopische Techniken eingesetzt (zum Teil auch handassistiert). Dabei sind allerdings gewisse Risiken und Probleme zu beachten (8). So liegen bei der laparoskopischen Nephrektomie sowohl die Rate des akuten Nierenversagens als auch die Zahl der Ureterverletzungen etwas höher. Letztendlich ist die Transplantatüberlebensrate und -funktion für beide Verfahren vergleichbar $(8,18)$. Im Allgemeinen gilt jedoch der konventionelle Flankenschnitt als sicherer, die laparoskopische Nierenentnahme zur Lebendspendetransplantation dagegen wird eher kritisch gesehen.

\section{Risiken für den Spender}

Wie bei jedem operativen Eingriff ist bei einer Nierenspende durchaus mit perioperativen Komplikationen

\section{Abb. 4 Fünf-Jahres-Transplantatüberleben nach Lebendspende} abhängig vom Spenderalter

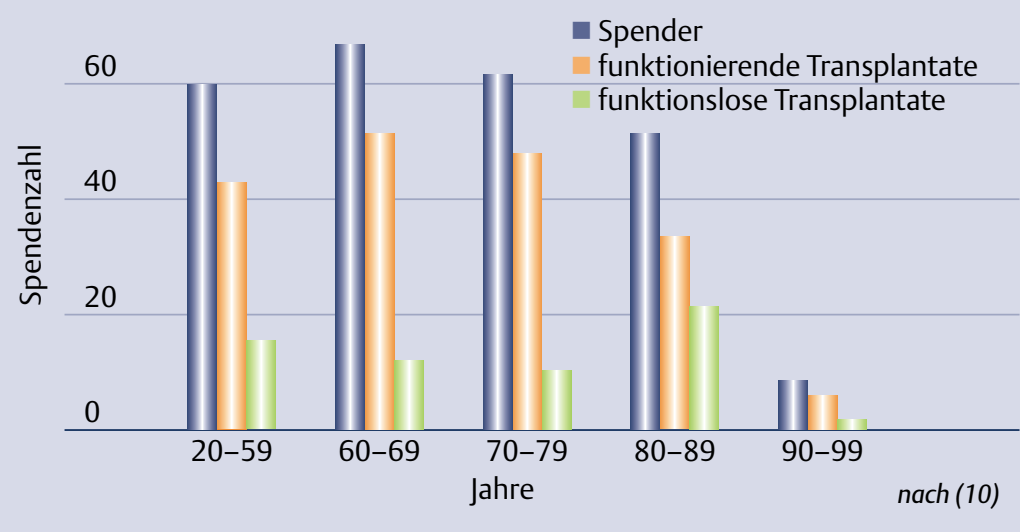

\section{Tab. 2 Spender-/Empfängerpaare im Transplantationszentrum} Düsseldorf (1997-2002)

Gesamtzahl untersuchter potenzieller Spender $n=109$ nicht akzeptierte bzw. nicht realisierte Spende und Transplantation $n=49$

Ursachen

- internistische Probleme beim Spender, neu diagnostiziert $\quad \mathrm{n}=13$ (koronare Herzerkrankung, Malignom, COPD)

- Angst des Spenders oder Empfängers nach Aufklärung $\quad n=13$

- Nierenerkrankung des Spenders

$\mathrm{n}=10$

- Zentrumswechsel

- immunologische Probleme zwischen Empfänger und Spender

$\mathrm{n}=6$

- Transplantation einer Leichenniere während der Vorbereitung - sonstiges

$\mathrm{n}=6$

$\mathrm{n}=3$

$\mathrm{n}=5$

realisierte Lebendspendetransplantationen zu rechnen. Die Analyse in Zentren mit langjähriger Erfahrung ergab diesbezüglich eine Mortalitätsrate von $0,03 \%$ bei Lebendnierenspenden (24). Weitere Komplikationen wie Blutungen, Pneumothorax, Infektionen oder Wunddehiszenz treten in einer Häufigkeit von 12-17\% auf (4). Aus den eigenen Erfahrungen bei über 100 Nierenspendeoperationen betrug die Spendermortalität bisher $0 \%$, die perioperative Komplikationsrate lag bei etwa $13 \%$ (25). Subjektiv berichten ungefähr 30\% der Spender, dass das Verfahren schmerzhaft und unangenehm war.

Langzeitbeobachtungen, die bis zu 25 Jahre nach einer Nierenspende umfassen, zeigen eine Verminderung der Kreatininclearance auf etwa 70\% des Ausgangswertes (9). Es sollte aber auch nicht unerwähnt bleiben, dass nach mehr als 20 Jahren vereinzelt auch Nierenspender die Dialysepflichtigkeit erreicht haben bzw. selber einer Nierentransplantation unterzogen wurden $(6$, 20). Eine Proteinurie von über 150 $\mathrm{mg} /$ Tag lag bei $10-20 \%$ der Spender vor. Die Hypertonie-Inzidenz betrug zwischen 35 und $40 \%$ und war vergleichbar mit der Inzidenz in einer altersangepassten Bevölkerung (7).

Ob sich die Komplikationsrate für den Spender durch die Entnahmetechnik wesentlich ändert, ist Gegenstand aktuellster Diskussionen. Hier ist der laparoskopische $\mathrm{Zu}$ gang im Vergleich zum offenen $\mathrm{Zu}$ gang zu erwähnen. Zumindest in Bezug auf den Gebrauch von Schmerzmitteln und die postoperative Mobilisation bietet die laparoskopische Nephrektomie sicherlich Vorteile gegenüber der bisher am meisten verbreiteten Technik des offenen $\mathrm{Zu}$ gangs $(15,26)$.

\section{Ergebnisse der Lebend- nierentransplantation}

Die Ergebnisse der Lebendnierenspende bzw. -transplantation zeigen exzellente Patienten- und Transplantatüberlebensraten, sowohl zwischen verwandten als auch nichtverwandten Spender-Empfänger-Paaren: So sind Fünf-Jahres-Transplantatüberlebensraten von $77 \%$ (Verwandte) und 79\% (Nichtverwandte) und Zehn-JahresTransplantatüberlebensraten von etwa 
60-70\% belegt. Leichennierentransplantate weisen im Gegensatz dazu eine Zehn-Jahres-Überlebensrate von ungefähr $45 \%$ auf $(3,4,19)$.

Erstaunlicherweise haben Nieren von nichtverwandten Spendern mit 15,5 Jahren eine längere Transplantathalbwertszeit als Nieren haploidentischer Lebendspender (10,8 Jahre) (19). Die Ergebnisse unseres Zentrums sind ähnlich (Abb. 3).

Ein Diskussionspunkt bei der Akzeptanz von Spendern ist immer wieder deren Alter. Mehrere Gruppen konnten aber bei Lebendspendetransplantationen mit älteren Spendern (über 65 Jahre) sehr gute Transplantationsergebnisse erzielen. Ein Beispiel der Ergebnisse aus einem Zentrum, bei der selbst über 70-80-Jährige eine Niere gespendet haben, zeigt die Abbildung 4 (10).

Der Anteil der Organe mit einem akuten Nierenversagen ist mit 6-10\% bei der Lebendnierentransplantation deutlich geringer als bei einer Leichennierentransplantation. Hier beträgt die Inzidenz immerhin zwischen 44 und 50\%. Die Zahl der Abstoßungen ist entweder ähnlich oder auch geringer im Vergleich zur Leichenspendetransplantation. Sie hängt jedoch auch von der Missmatch-Konstellation ab. Bei nichtverwandten Transplantationen, bei denen in der Regel eine höhere Immunsuppression erfolgt, ist die Zahl der infektiösen Komplikationen unter Umständen höher. Ein Vergleich der Ergebnisse zwischen verwandten und nichtverwandten Transplantationen, die in der Zeit zwischen 1997 und Juli 2001 in unserem Zentrum durchgeführt wurden, werden in Tabelle 3 dargestellt (25).

\section{Perspektiven}

In Anbetracht der langen Wartezeiten auf ein passendes Organ von einem Verstorbenen, und unter Berücksichtigung der exzellenten Ergebnisse der Lebendnierenspende und -transplantation erscheint es gerechtfertigt, diese Möglichkeit jedem Patienten vorzuschlagen - am besten sogar noch bevor dieser das Stadium der Dialysepflichtigkeit erreicht. Aus unserer Sicht wäre die Lebendnierenspende und -transplantation eindeutig zu propagieren.

\begin{tabular}{|llll}
\hline Tab. 3 & $\begin{array}{l}\text { Vergleich von verwandten und nichtverwandten } \\
\text { Nierentransplantationen (Düsseldorf, 1997-2001) }\end{array}$ \\
\hline & $\begin{array}{l}\text { verwandte } \\
(\mathbf{n = 3 8 )}\end{array}$ & $\begin{array}{l}\text { nichtverwandte } \\
(\mathbf{n = 2 4 )}\end{array}$ & $\mathbf{p}$ \\
\hline akutes Nierenversagen & $6(15,8 \%)$ & $1(4,2 \%)$ & $\mathrm{ns}$ \\
\hline Abstoßungen (Patienten) & $20(52,5 \%)$ & $13(54,2 \%)$ & $\mathrm{ns}$ \\
\hline infektiöse Komplikationen & $14(36,8 \%)$ & $16(66,7 \%)$ & 0,036 \\
\hline medizinische Komplikationen & $9(23,7 \%)$ & $7(29,2 \%)$ & $\mathrm{ns}$ \\
\hline Kreatinin (mg/dl) & $1,76 \pm 0,6$ & $1,62 \pm 0,5$ & $\mathrm{~ns}$ \\
\hline Transplantatüberleben & $94,8 \%$ & $100 \%$ & $\mathrm{~ns}$ \\
\hline Patientenüberleben & $97,4 \%$ & $100 \%$ & $\mathrm{~ns}$ \\
\hline
\end{tabular}

Unabhängig davon darf dabei die Förderung der postmortalen Organspende unter keinen Umständen nachlassen.

Ein in Deutschland mehrfach diskutierter Punkt ist die Erweiterung des Spenderpools durch so genannte Crossover-Transplantationen. In diesem Fall werden Spende und Transplantation zwischen zwei Paaren „über Kreuz“ durchgeführt. Eine solche Konstellation ist vorstellbar, wenn eine Nierenspende aufgrund einer ABO-Blutgruppeninkompatibilität nicht zustande kommen kann und ein anderes Paar mit der gleichen Problematik existiert. Durch die gleichzeitige Spende über Kreuz wäre das Problem gelöst. Die Voraussetzungen, die das Transplantationsgesetz hierzu vorgibt (z.B. besondere Verbundenheit und Ausschluss des Organhandels), können diesbezüglich unterschiedlich ausgelegt werden. Letztendlich ist die Handhabung einer Crossover-Situation in der deutschen Rechtslehre umstritten, eine Klärung durch die Rechtsprechung steht aus.

Eine insbesondere in den USA geführte aktuelle Diskussion betrifft die altruistische, ungerichtete Lebendspende etwa in einen Organpool ( 1 , 14, 22). Eine solche Nierenspende ist jedoch in Deutschland aufgrund des Transplantationsgesetzes nicht möglich. Mit seiner Entscheidung vom August 1999 hat das Bundesverfassungsgericht Einwände gegen dieses Verbot zurückgewiesen.

Kürzlich wurde die Bezahlung einer Nierenspende aus ökonomischer Hinsicht als eine weitere Möglichkeit zur Verminderung des Organmangels diskutiert. In Deutschland ist dies jedoch nicht zulässig und strafbar. Strafbar ist auch die Transplantation oder der Erwerb kommerziell erworbener Nieren, was in manchen anderen Ländern möglich ist. Nicht nur aus juristischer sondern auch aus medizinischer Sicht ist davor jedoch aufgrund zahlreicher, vor allem infektiöser Komplikationen - zu warnen.

Letztendlich muss nochmals auf die „Subsidiarität“ der Lebendspende hingewiesen werden, welche die höhere Priorität der postmortalen Nierentransplantation bedingt. Bei allen Bemühungen, die Lebendspende zu aktivieren, muss die Förderung der Leichennierenspende mit vielfachen, bisher immer noch nicht ausreichend ausgeschöpften Möglichkeiten absoluten Vorrang haben.

\section{Living Kidney Donation and Trans- plantation - High Long-term Graft Survival and Low Risks} In view of low and stagnant rates of kidney transplantations with organs from cadaveric donors, clear legal regulations and excellent results of living kidney transplantations, there has been an increasing number of transplantations with kidneys from living donors in Germany during the last years. Related and nonrelated persons with close personal relationships can present as potential donors. Further requirements for a living donation are voluntariness, exclusion of commercial interests, bloodgroup compatibility, a negative cross-match and absence from severe disease. Psychological and ethical considerations are also essential. Living kidney donation and transplantation as well between related as 
Ins Netz gegangen

http://www.nephrologie.de

Die Homepage der Deutschen Arbeitsgemeinschaft für Klinische Nephrologie bietet vielfältige Informationen. Natürlich können Sie sich hier über die Ziele der Gesellschaft oder ihre Projekte informieren. Außerdem finden Sie verschiedenste Fachinformationen, wie zum Beispiel Richtlinien rund um die Transplantationsmedizin, einen Veranstaltungskalender und eine umfangreiche Link-Liste.

\section{http://www.gfn-online.com}

Sie suchen Informationen zu nationalen oder internationalen nephrologischen Fachgesellschaften oder zu Themen wie Dialyse bzw. Nierentransplantation? Dann sind Sie auf der Homepage der Gesellschaft für Nephrologie gut aufgehoben. Hier können Sie sich aber auch über aktuelle klinische Studien informieren, die zurzeit an deutschen Universitäten laufen - nicht nur in der klassischen Nephrologie, sondern zum Beispiel auch bei Hochdruckerkrankungen. Über die gut gegliederte Aufstellung zu verschiedenen Nephrologie-Links können Sie zudem direkt die Homepages der Transplantationszentren in Deutschland erreichen.

\section{http://www.hochdruckliga.info}

An Ärzte und an Patienten wendet sich die Deutsche Liga zur Bekämpfung des hohen Blutdruckes - Deutsche Hypertonie Gesellschaft e.V. mit ihrer Homepage. Ob Sie sich über die HypertonieLeitlinien, Therapieempfehlungen oder internationale Fachgesellschaften informieren oder Angebote für die Fortbildung nutzen wollen, hier finden Sie sich sicherlich zurecht. Die Hochdruckliga bietet beispielsweise Bildvorlagen für Arzt-Patientenseminare. Online abrufen können Sie zudem patientengerechte Merkblätter und Empfehlungen (z.B. Hypertonie und Sport, Alkohol und Bluthochdruck). Im Servicebereich für Patienten bietet die Hochdruckliga aber auch Informationen rund um Selbsthilfegruppen. Und am HerzKreislauf-Telefon beantworten Experten alle Fragen zur Hypertonie. between non-related persons offer several advantages, such as optimal preparation and excellent short- and longterm survival of the grafts. The risks for the donor are low but not negligable and detailed informed consent is mandatory. In our view, living kidney donation should be promoted, e.g. by expanding the number of preemptive transplantations and by inclusion of elderly persons, but cadaveric kidney donation needs even more intensive support.

\section{Key Words}

kidney failure - living kidney donation - cadaveric kidney donation graft survival - legal regulations

\section{Literatur}

1. Adams PL, Cohen DJ, Danovitch GM et al. The nondirected live-kidney donor: ethical considerations and practice guidelines: A National Conference Report. Transplantation 2002; 74: 582-589

2. Asderakis A, Augustine T, Dyer $P$ et al. Pre-emptive kidney transplantation: the attractive alternative. Nephrol Dial Transplant 1998; 13: 1799-1803

3. Cortesini R, Pretagostini R, Bruzzone P, Alfani D. Living unrelated kidney transplantation. World J Surg 2002; 26: 238-242

4. D'Alessandro AM, Sollinger HW, Knechtle SJ et al. Living related and unrelated donors for kidney transplantation. A $28-$ year experience. Ann Surg 1995; 222: 353-362; discussion 362-364

5. DSO. DSO Jahresbericht 2002

6. Ellison MD, McBride MA, Taranto SE et al. Living kidney donors in need of kidney transplants: a report from the organ procurement and transplantation network. Transplantation 2002; 74: 1349-1351

7. Fehrman-Ekholm I, Duner F, Brink B et al. No evidence of accelerated loss of kidney function in living kidney donors: results from a cross-sectional follow-up. Transplantation 2001; 72: 444-449

8. Gershbein AB, Fuchs $G$ J. Hand-assisted and conventional laparoscopic live donor nephrectomy: a comparison of two contemporary techniques. J Endourol 2002; 16: 509-513

9. Goldfarb DA, Matin SF, Braun WE et al. Renal outcome 25 years after donor nephrectomy. JUrol 2001; 166: 2043-2047 10. Hadjiyannakis EJ, Hadjidimitriou F, Drakopoulos $S$ et al. Renal transplantation from older living donors. Transplant Proc 2001; 33: 906-908

11. Humar A, Durand B, Gillingham K et al. Living unrelated donors in kidney transplants: better long-term results than with non-HLA-identical living related donors? Transplantation 2000; 69: 1942-1945

12. Kasiske BL, Snyder J], Matas AJ et al. Preemptive kidney transplantation: the advantage and the advantaged. J Am Soc Nephrol 2002; 13: 1358-1364
13. Koo DD, Welsh KI, McLaren AJ et al. Cadaver versus living donor kidneys: impact of donor factors on antigen induction before transplantation. Kidney Int 1999; 56: 1551-1559

14. Landolt MA, Henderson AJ, Barrable WM et al. Living anonymous kidney donation: what does the public think? Transplantation 2001; 71: 1690-1696

15. Lennerling A, Blohme I, Ostraat $O$ et al. Laparoscopic or open surgery for living donor nephrectomy. Nephrol Dial Transplant 2001; 16: 383-386

16. Meier-Kriesche HU, Kaplan B. Waiting time on dialysis as the strongest modifiable risk factor for renal transplant outcomes: a paired donor kidney analysis. Transplantation 2002; 74: 1377-1381

17. Najarian JS, Chavers BM, McHugh LE, Matas AJ. 20 years or more of follow-up of living kidney donors. Lancet 1992; 340: 807-810

18. Nogueira JM, Cangro CB, Fink JC et al. A comparison of recipient renal outcomes with laparoscopic versus open live donor nephrectomy. Transplantation 1999; 67: 722-728

19. Opelz G. CTS-Daten. 2002

20. Ramcharan T, Matas AJ. Long-term (20-37 years) follow-up of living kidney donors. Am J Transplant 2002; 2: 959-964

21. Saunders RN, Elwell R, Murphy G et al. Workload generated by a living donor programme for renal transplantation. Nephrol Dial Transplant 2000; 15: 1667-1672

22. Spital A. Public attitudes toward kidney donation by friends and altruistic strangers in the United States. Transplantation 2001; 71: 1061-1064

23. Terasaki PI, Cecka JM, Gjertson DW, Takemoto S. High survival rates of kidney transplants from spousal and living unrelated donors. N Engl J Med 1995; 333: 333-336

24. Valente JF, Hariharan S, Peddi VR et al. Causes of renal allograft loss in black vs. white transplant recipients in the cyclosporine era. Clin Transplant 1997; 11: 231-236 25. Voiculescu A, Ivens K, Hetzel GR et al. Kidney transplantation from related and unrelated living donors in a single German centre. Nephrol Dial Transplant 2003; 18: 418-425

26. Waller JR, Hiley AL, Mullin EJ et al. Living kidney donation: a comparison of laparoscopic and conventional open operations. Postgrad Med J 2002; 78: 153-157

\section{Anschrift für die Verfasser}

Dr. Adina Voiculescu

Medizinische Klinik und Poliklinik

Klinik für Nephrologie und Rheumatologie Universitätsklinikum Düsseldorf Heinrich-Heine-Universität Düsseldorf Moorenstr. 5

40225 Düsseldorf 